\title{
LA REFORMA DEL FONDO DE COMPENSACIÓN INTERTERRITORIAL. LOS COSTES Y BENEFICIOS DE LA SOLIDARIDAD
}

Luis Ángel HIERRO RECIO *

\section{EL FONDO DE COMPENSACIÓN INTERTERRITORIAL EN EL PERÍODO TRANSITORIO.}

El proceso de definición del sistema de financiación autonómica ha atravesad diversos períodos, el que abarca hasta 1983, caracterizado por ser el período d aprobación de los distintos Estatutos y en el que estaban aún pendientes d concreción algunos aspectos del llamado "sistema transitorio" de financiación. U segundo período, en el que se mantiene vigente dicho sistema transitorio y qu comprende los años 1984 a 1986, ambos incluidos. Un tercer período, 1987-199? en el que se concreta la financiación autonómica según el llamado "sistem definitivo", con pequeñas transformaciones incluidas en 1992. Y el cuartı período que se abre en 1994, con la cesión del 15\% de la recaudación del IRPF

La base del sistema transitorio se encontraba en el concepto de coste efectivc y en la asociación que se establecía entre los distintos componentes de coste : los métodos de financiación alternativos. Así, el coste de un servicio s descomponía en:

a) Coste efectivo:

- Gastos de personal.

- Compra de bienes corrientes y servicios.

- Inversiones de reposición.

*. Profesor Titular del Departamento de Teońa Económica y Economía Política de la Universidad d Sevilla. 
b) Coste complementario:

- Inversiones nuevas.

- Subvenciones corrientes.

- Subvenciones de capital.

y la financiación asociada se establecía de la siguiente forma:

a) Para el coste efectivo:

- Tributos cedidos.

- Participación en los ingresos del Estado.

b) Para el coste complementario:

- Fondo de Compensación Interterritorial.

- Transferencias directas de los Presupuestos Generales del Estado.

De manera que la participación en ingresos se calculaba en base al coste efectivo de los servicios y el FCI se utilizaba para financiar inversiones nuevas. Así, el segundo gran pilar sobre el que se sustentó la financiación autonómica fue el Fondo de Compensación Interterritorial. Dicho fondo ya fue introducido en los Presupuestos Generales de 1982, pero en realidad su verdadera incidencia en la financiación autonómica no empieza a contar hasta 1984, año en que se aprobó su Ley reguladora ${ }^{1}$.

En origen, el FCI se concibió como la manifestación fundamental del principio de solidaridad interregional, teniendo como misión minorar los desequilibrios económicos interterritoriales. Su cuantía mínima venía determinada por el $30 \%$ de la inversión pública, definida como inversión real nueva ${ }^{2}$. Quiere ello decir, que en las dotaciones del fondo no participaba la inversión de reposición, que iba incluida en el coste efectivo de los servicios transferidos.

La distribución del fondo se realizaba de la siguiente forma:

a) Todas la Comunidades Autónomas eran beneficiarias del FCI, debiendo cada una de ellas elaborar un programa de desarrollo regional que serviría de base para las inversiones a financiar con la participación en el FCl.

b) La distribución del fondo se realizaba asignando a cada Comunidad un porcentaje obtenido en un sistema de tramos, que se asociaba a variables definidoras de las diferencias interregionales.

1. En 1982 la totalidad del FCI fue gestionada por la Administración Central y en 1983 sólo $16,2 \%$ de la cuantía del FCI fue gestionada por las Comunidades Autónomas.

2. Una discusión amplia sobre el tema del porcentaje puede verse en GARCÍA AÑOVEROS (1986a, 1986b). 
c) Calculados dichos porcentajes de participación, y dado que se valoraba e "hecho insular" como definidor de necesidades adicionales para Canaria y Baleares, se incrementaba el porcentaje de estas comunidades en un $5 \%$ más un $1 \%$ adicional por cada $50 \mathrm{Km}$. de distancia entre los territorio insulares y la península. Procediéndose con posterioridad a minorar dichi cantidad en el porcentaje de las Comunidades no insulares, de maneri proporcional.

El resultado era que el FCI se repartía en un $70 \%$ inversamente proporciona a la renta per cápita, ponderada por el número de habitantes de cada región; et un $20 \%$ proporcionalmente al saldo migratorio, considerando tanto la migracióı interna como la externa de los últimos 10 años; en un $5 \%$ proporcionalmente a desempleo diferencial respecto a la media; y en un $5 \%$ proporcional a li superficie del territorio. Con la particularidad de que en el segundo tramo sók participaban las Comunidades con saldo migratorio positivo, y en el tercer tramc sólo lo hacían las Comunidades en que el desempleo fuese superior a la media

A lo largo de los años del período transitorio se dedicaron anualmente al FC. unos 200.000 millones de pesetas, por término medio. Siendo significativc destacar que en todos los años fue aumentando el porcentaje de dicho fondc gestionado por las Comunidades Autónomas, que ya en 1986 alcanzó el 74,2\% de la dotación del fondo. Otro dato importante de reseñar es la asignación de lı financiación obtenida del FCI, pues representó un volumen muy importante d€ ingresos para las Comunidades Autónomas. A título de ejemplo pueden tomars los datos de 1985, año en el que los ingresos del FCI incrementaron Í financiación autónoma en un $35 \%$ sobre el coste efectivo de los servicios transferidos ${ }^{3}$. Evidentemente ello era consecuencia de que en realidad el FC] estaba cumpliendo una misión de suficiencia financiera, más que su objetivo de instrumentación de la solidaridad interregional.

\section{EL SISTEMA DE FINANCIACIÓN DEFINITIVO.}

En 1987 entró en vigor el que se denominó sistema "definitivo" de financiación autonómica, que había sido aprobado en noviembre de 1986 por e] Consejo de Política Fiscal y Financiera.

3. Véase TORRES COBO (1984). 
El sistema definitivo se creó con los objetivos de: permitir financiación suficiente a las Comunidades Autónomas, preservar la solidaridad y hacer compatibles las políticas económicas del Estado con la gestión de las Comunidades Autónomas. Y para ello se partió de la concepción de que debían existir dos tipos o bloques de financiación:

- La financiación "incondicionada", que tenía como objetivo garantizar la suficiencia financiera de las Comunidades Autónomas y la autonomía de las mismas en su gasto.

- Y la financiación "finalista", que se caracterizaba por estar constituida por asignaciones de fondos cuyo uso estaba condicionado a la aplicación a finalidades concretas.

En realidad, esta diferencia ya aparecía implícita en la estructura del sistema transitorio. La particularidad se encontraba en que con el sistema definitivo desaparecía el concepto de coste efectivo, asumiendo los fondos de financiación incondicionada el objetivo de financiar tanto los componentes de gasto que antes se consideraban como coste efectivo, como el gasto en inversión nueva y parte de las transferencias asociadas a los servicios transferidos. Permitiéndose así plena autonomía para que cada Comunidad distribuyese su gasto entre cualesquiera de los tres tipos citados, y eliminando el problema que había creado el sistema transitorio con la inversión nueva.

Este nuevo sistema supuso una modificación del FCI, ya que al pasar la financiación de la inversión nueva a ser responsabilidad del bloque de financiación incondicionada, el FCI dejaba de tener como objetivo la suficiencia financiera para asumir la obligación de ser el principal sustentador del principio de solidaridad. Configurando, junto con las transferencias condicionadas específicas, el bloque de la financiación finalista.

Evidentemente este traspaso de atribuciones hacia la financiación incondicionada llevaba aparejada una modificación en la cuantía del FCI, quedando incorporados el $25 \%$ de los fondos, que hasta 1986 habían sido repartidos por esta vía, a la financiación incondicionada. Ello supuso que a partir de 1987, el FCI sólo estaba dotado con un $30 \%$ de los fondos destinados a inversión por parte del Estado, frente al $40 \%$ anterior. A cambio, se estableció un mecanismo de coordinaición entre el FCI y los fondos FEDER, de manera que cualquier Comunidad podía cofinanciar proyectos de inversión con los fondos comunitarios recibidos por España, con un límite máximo del 30\% de la cantidad recibida por la comunidad vía $\mathrm{FCI}^{4}$.

4. Para un tratamiento específico del FCl en el sistema definitivo véase MELGUIZO (1988) y UTRILLA (1991). 
A mediados de 1989 se alcanzó un acuerdo de modificación del FCI qu alteró en gran medida su concepción. Por una parte, se modifica su estructura de forma que a partir de 1990 sólo reciben fondos vías FCI las Comunidade Autónomas cuya renta per cápita sea inferior al $75 \%$ de la renta per cápit Europea. Esta modificación hace coincidir los criterios utilizados por el FCI col los criterios establecidos por la Comunidad Europea para la selección de lo beneficiarios de los fondos estructurales, y elimina del reparto a las siguiente Comunidades: Cataluña, País Vasco, Madrid, Aragón, Cantabria, Baleares, L. Rioja y Navarra.

Consecuencia evidente de este cambio son las siguientes transformaciones:

- Se modifican las ponderaciones de los criterios de reparto del FCI, qu pasan a ser las del cuadro.

\begin{tabular}{|l|c|c|}
\hline \multicolumn{1}{|c|}{ CRITERIOS } & FCI-1984 & FCI-I990 \\
\hline Población & $70 \%$ & $87,5 \%$ \\
\hline Superficie & $5 \%$ & $3 \%$ \\
\hline Desempleo & $5 \%$ & $1 \%$ \\
\hline Saldo migratorio & $20 \%$ & $1,6 \%$ \\
\hline Dispersión de poblac. & - & $6,9 \%$ \\
\hline
\end{tabular}

- En segundo lugar se introducen dos correcciones posteriores ya que si incrementa el fondo en un $23,21 \%$, que se reparte: el $20 \%$ inversament a la renta per cápita y el 3,21\% de dotación a Canarias por insularidad.

- Finalmente, y en tercer lugar, se disminuye la cuantía del FCI, que sigu manteniéndose en un $30 \%$ de la inversión civil nueva, pero ponderado po la población de las Comunidades Autónomas que son beneficiarias. E decir, en realidad el porcentaje deja de ser el $30 \%$.

Es claro que una transformación tan profunda del FCI, que excluye a mucha Comunidades de su participación, no tenía viabilidad si a cada Comunidad no st le dotaba por otra vía los fondos que antes recibía de FCI. Es por eso por lo qu a partir de 1990 se concede una compensación de carácter transitorio destinad a cubrir los gastos de inversión que antes financiaba el FCI, que tiene una cuantíi 
prácticamente igual a la dotación del FCI y que hace que en realidad sea escasa la repercusión de la modificación del fondo. Más, cuando por acuerdo del Consejo de Política Fiscal y Financiera, de 20 de enero de 1992, se integra la Compensación Transitoria en el bloque de la financiación incondicionada.

\section{LA SOLIDARIDAD EN LA FINANCIACIÓN AUTONÓMICA.}

Lo anterior no es más que una manifestación del verdadero problema del sistema definitivo de financiación, que no es otro que la existencia de gran número de Comunidades que no están de acuerdo con los resultados redistributivos del sistema. $\mathrm{Y}$ es en este punto donde entra en la discusión la noción de solidaridad.

La "solidaridad", incluida en la Constitución como principio informador deI sistema de financiación autonómica, puede ser, y de hecho es, un concepto del que caben tantas interpretaciones como especialistas se dedican a su estudio, pues es la manifestación de los juicios de valor de quien la define. Quiere esto decir, que cuando se intenta diseñar un sistema de financiación altemativo, cada autor en función a su concepción de solidaridad incluye en su propuesta los medios destinados a hacer efectivo dicho principio.

Una forma de aclarar la cuestión es distinguir entre los distintos contenidos que se le pueden dar a la noción de solidaridad. Así, un primer contenido sería que la solidaridad debe garantizar que los sujetos tengan igualdad de acceso a los servicios prestados por las Comunidades. En este caso estaríamos considerando un componente de "nivelación de servicios", que a su vez tendría dos subcomponentes: uno relativo a que todas las Comunidades deben disponer de dotaciones similares de infraestructuras y capital público, "nivelación de infraestructuras"; y otro referido a que las Comunidades deben tener capacidad de ofrecer los mismos servicios atendiendo a las necesidades y con independencia de las disparidades de costes que tengan que soportar, "nivelación por diferencias en servicios".

Otra posible interpretación de la solidaridad es la que considera que el sistema de financiación debe evitar las diferencias en ingresos entre Comunidades. Es decir, dado que manteniendo idéntico sistema impositivo la distribución de bases entre Comunidades no es homogénea, es necesario garantizar que el sistema provea uniformemente de fondos en términos de recursos per cápita. Estaríamos en este caso en una interpretación de la solidaridad como garante de la "igualación en la capacidad de gasto".

Finalmente, cabe la posibilidad de dotar de contenido a la solidaridad considerando que la misma constituye una manifestación de la necesidad de 
igualar la capacidad productiva de las distintas regiones. Es decir se puec interpretar la solidaridad como la garantía del desarrollo equilibrado de todo , territorio nacional. En este sentido diremos que existe un componente c "redistribución para el desarrollo" 5.

Sea cual sea la componente interpretativa de la solidaridad, su instrumentacić siempre va a producir dos efectos antagónicos: un beneficio para la Comunida que resulte como "desfavorecida" por la interpretación utilizada, que recibirá unc fondos adicionales; y un perjuicio para la que aparezca como región "privileg ada", ya que como poco dejará de recibir esos fondos. Pero la valoración c región beneficiada o perjudicada, no sólo dependerá de la posición que ocupas con anterioridad a la implantación del nuevo sistema. Es decir hay que distingu entre beneficio y perjuicio absoluto, y beneficio y perjuicio relativo respecto $\mathrm{d}$ la situación anterior.

Además, como toda renegociación de la financiación vía fondos se plante inicialmente en forma de juego de suma nula, donde unos pierden para que otrc ganen, es evidente que los perdedores, salvo que mantengan una postura altruist que entendemos de dudosa existencia, se negarán a la modificación. Y 1 solución para sus aceptación pasará por transformar el problema en un juego d suma positiva en el que todos ganen, aunque unos lo hagan más que otros.

\section{EL COSTE DE LA SOLIDARIDAD.}

En realidad, la reforma de 1989 sólo supuso un leve retoque en la concepció del FCI, pues al final, y como ocurrió en ocasiones anteriores, el FCI sirvió má de elemento de ajuste de la financiación autonómica, que de mecanismo d articulación de la solidaridad interterritorial.

Prueba de ello fue:

- La introducción de la ponderación de la población implicada, a la hora d definir el montante del FCI como el $30 \%$ de la inversión civil nueva.

- La aparición de la llamada Compensación Transitoria, que ascendía a un cuantía similar a la del propio FCI y que se repartía entre todas la Comunidades.

- Y finalmente, el hecho de que se alterasen levemente las ponderaciones d los criterios que servían para definir el porcentaje de participación en e

5. Cabe una cuarta interpretación de la solidaridad como garante de la "redistribución de la renta de lc sujetos", sin embargo no parece acertado su tratamiento por múltiples motivos jurídicos y económicos. 
FCI, estableciéndose además cuantías específicas adicionales a repartir con criterios distintos.

En definitiva, en aras de garantizar que la reforma del sistema de financiación de transformarse en un juego de suma positiva, se desaprovechó la oportunidad de articular el FCI en base a una concepción determinada del principio de solidaridad, a la par que se aumentó la complejidad del mismo, enmascarando la manifiesta insolidaridad derivada de la evolución del sistema, a través del artificio de la Compensación Transitoria.

Parte de los resultados del proceso que acabo de describir se ponen de manifiesto en los cuadros I y II, donde se representan las cifras asignadas en los Presupuestos Generales del Estado de los ejercicios 87, 88, 89, 90, 91 y 92 a cada Comunidad en concepto de FCI, para los tres primeros años, y de FCI más Compensación Transitoria, para los tres últimos, así como los porcentajes correspondientes sobre el total de la dotación.

\section{CUADRO I}

\begin{tabular}{|c|c|c|c|c|c|c|}
\hline \multirow{2}{*}{$\frac{\text { F.C.I. }}{\text { C.A. }}$} & \multicolumn{2}{|c|}{1987} & \multicolumn{2}{|c|}{1988} & \multicolumn{2}{|c|}{1989} \\
\hline & Mill. pts & $\%$ part. & Mill. pts. & $\%$ part. & Mill. pts. & $\%$ part. \\
\hline Andalucía & $35.771,40$ & 25,53 & $36.927,60$ & 24,55 & $49.855,60$ & 24,47 \\
\hline Aragón & $3.069,10$ & 2,19 & $2.904,90$ & 1,93 & $4.269,20$ & 2,10 \\
\hline Asturias & $3.454,60$ & 2,47 & $3.491,40$ & 2,32 & $4.763,30$ & 2,34 \\
\hline Baleares & $1.177,60$ & 0,84 & $1.031,40$ & 0,69 & $1.541,90$ & 0,76 \\
\hline Canarias & $7.183,00$ & 5,13 & $8.745,40$ & 5,81 & $11.750,50$ & 5,77 \\
\hline Cantabria & $1.324,60$ & 0,95 & $1.201,10$ & 0,80 & $1.767,30$ & 0,87 \\
\hline Castilla León & $12.717,20$ & 9,08 & $11.498,00$ & 7,64 & $16.118,10$ & 7,91 \\
\hline Cas.-La Mancha & $10.579,20$ & 7,55 & $10.813,70$ & 7,19 & $15.047,30$ & 7,38 \\
\hline Cataluña & $10.044,70$ & 7,17 & $14.768,80$ & 9,82 & $23.892,50$ & 11,73 \\
\hline Valencia & $8.744,30$ & 6,24 & $9.495,10$ & 6,31 & $13.490,10$ & 6,62 \\
\hline Extremadura & $11.288,00$ & 8,06 & $9.366,20$ & 6,23 & $12.739,60$ & 6,25 \\
\hline Galicia & $15.383,70$ & 10,98 & $16.297,40$ & 10,83 & $22.889,70$ & 11,23 \\
\hline Madrid & $7.774,20$ & 5,55 & $8.637,00$ & 5,74 & $12.477,50$ & 6,12 \\
\hline Murcia & $2.710,20$ & 1,93 & $3.271,20$ & 2,17 & $5.071,10$ & 2,49 \\
\hline Navarra & 999,40 & 0,71 & 982,30 & 0,65 & $1.458,40$ & 0,72 \\
\hline País Vasco & $7.435,60$ & 5,31 & $10.543,30$ & 7,01 & $5.994,00$ & 2,94 \\
\hline La Rioja & 473,50 & 0,34 & 460,50 & 0,31 & 635,00 & 0,31 \\
\hline TOTAL & $140.130,3$ & 100,00 & $150.435,30$ & 100,00 & $203.761,10$ & 100,00 \\
\hline
\end{tabular}

Fuente: Presupuestos Generales del Estado y elaboración propia. 
Vistas las mencionadas cifras, los objetivos propuestos son dos:

- $\quad$ En primer lugar, determinar y valorar los costes o beneficios financierc obtenidos por cada Comunidad Autónoma, como consecuencia de evolución del sistema aplicado en el FCI en los últimos años.

$\mathrm{Y}$ en segundo lugar, determinar esos hipotéticos costes y beneficio partiendo del supuesto de que se hubiese diseñado el FCI en la base criterio de interpretar la solidaridad como "redistribución para el desarrr $10^{\prime}$ y, por ende, como una contribución a maximizar las producciones pt cápita de las regiones menos desarrolladas.

CUADRO II

\begin{tabular}{|c|c|c|c|c|c|c|}
\hline \multirow{2}{*}{$\frac{\text { F.C.I. + C.T. }}{\text { C.A. }}$} & \multicolumn{2}{|c|}{1990} & \multicolumn{2}{|c|}{1991} & \multicolumn{2}{|c|}{1992} \\
\hline & Mill. pts & $\%$ part. & Mill. pts. & $\%$ part. & Mill. pts. & $\%$ par \\
\hline Andalucía & $65.768,00$ & 27,43 & $71.203,90$ & 27,66 & $58.935,00$ & 27,4 \\
\hline Aragón & $4.538,00$ & 1,89 & $4.868,60$ & 1,89 & $4.071,70$ & 1,90 \\
\hline Asturias & $4.316,00$ & 1,80 & $5.150,20$ & 2,00 & $5.095,20$ & $2,3:$ \\
\hline Baleares & $1.613,00$ & 0,67 & $1.730,80$ & 0,67 & $1.443,90$ & $0,6:$ \\
\hline Canarias & $14.037,00$ & 5,85 & $14.471,70$ & 5,62 & $10.016,50$ & $4,6 t$ \\
\hline Cantabria & $1.831,00$ & 0,76 & $1.964,20$ & 0,76 & $1.638,70$ & $0,7 \mathrm{t}$ \\
\hline Castilla León & $18.315,00$ & 7,64 & $19.345,20$ & 7,52 & $17.544,10$ & $8,1^{\prime}$ : \\
\hline Cas.-La Mancha & $15.943,00$ & 6,65 & $17.064,20$ & 6,63 & $14.234,20$ & $6,6:$ \\
\hline Cataluña & $26.282,00$ & 10,96 & $28.259,80$ & 10,98 & $23.576,20$ & 10,98 \\
\hline Valencia & $14.621,00$ & 6,10 & $15.097,90$ & 5,87 & $12.185,80$ & 5,6 \\
\hline Extremadura & $15.515,00$ & 6,47 & $16.455,10$ & 6,39 & $14.195,90$ & 6,61 \\
\hline Galicia & $27.547,00$ & 11,49 & $30.025,20$ & 11,67 & $25.715,40$ & 11,98 \\
\hline Madrid & $12.066,00$ & 5,03 & $12.950,00$ & 5,03 & $10.803,80$ & 5,0 \\
\hline Murcia & $5.179,00$ & 2,16 & $5.645,50$ & 2,19 & $4.309,50$ & 2,01 \\
\hline Navarra & 0,00 & 0,00 & 0,00 & 0,00 & 0,00 & $0,0 c$ \\
\hline País Vasco & $11.556,00$ & 4,82 & $12.425,90$ & 4,83 & $10.366,50$ & $4,8:$ \\
\hline La Rioja & 675,00 & 0,28 & 724,30 & 0,28 & 604,30 & $0,2 \varepsilon$ \\
\hline TOTAL & $239.802,00$ & 100,00 & $257.382,50$ & 100,00 & $214.736,70$ & 100,00 \\
\hline
\end{tabular}

Fuente: Presupuestos Generales del Estado y elaboración propia.

Para conseguir el primer objetivo se propone tomar como base los porcentaje de participación de las Comunidades en el FCI en 1987 y calcular los costes beneficios financieros de las Comunidades de cada año, actualizándolos a pesetc de 1992. Es decir, se calculan las participaciones en el FCI suponiendo que $s$ 
hubiese mantenido constante el porcentaje de participación que cada Comunidad tenía en el año 1987, y se restan de las participaciones reales. En base a ello, podemos definir un Saldo Financiero Acumulado (hipótesis 1) para cada Comunidad, que expresa la cantidad de financiación "ganada" o "perdida en el período, y que viene dado por la ecuación:

$$
S F A=\sum_{i=1988}^{1992}[P P F C I(i)-P P F C I(1987)] \cdot F C I(i) \cdot[1+s(i)]
$$

donde s (i) es la inflación acumulada desde el año "i" hasta $1992^{6}$.

Más difícil es intentar conseguir el segundo objetivo, pues la cuantificación del saldo financiero para cada Comunidad es subjetiva y, por tanto, criticable. En concreto, en este trabajo se propone que la solidaridad interterritorial sea entendida como un principio que oriente la actividad financiera asociada al FCI hacia la obtención de un "desarrollo uniforme" de las Comunidades Autónomas.

La opción por dicha alternativa conduce a valorar que la magnitud sobre la que ha de construirse el sistema de reparto sea el Producto Interior Bruto o el Producto Nacional Bruto de cada Comunidad, en términos per cápita. Cabría, claro está, recurrir a algún concepto de Renta, sin embargo ello conduciría a error a la hora de valorar el nivel de desarrollo, pues en el mejor de los casos se estarían excluyendo las subvenciones de las Administraciones a la producción y en el peor de ellos, se estarían incorporando además las políticas redistributivas.

Ahora bien, dado que el interés se encuentra en asimilar los niveles de desarrollo regional, parece razonable que se corrija la magnitud de producción tomada, para que la ayuda al desarrollo se realice en proporción a la distancia que separa a cada Comunidad de la más desarrollada en cada momento ${ }^{7}$. De forma que cuanto mayor sea esa diferencia más participación se obtenga el en FCI.

6. Se toma el año 1987 como base por ser el primer ejercicio en el que el coste complementario de los servicios deja de ser financiado con el FCI y, por consiguiente, se puede considerar que a partir de ese año dicho fondo tiene asumida, exclusivamente, la función de articular la solidaridad interregional. Además, y a efectos del cálculo de la inflación acumulada, hay que señalar que las dotaciones de cada ejercicio se consideran referidas a comienzos de cada año.

7. Téngase en consideración que elegir la más desarrollada y no una media o cualquier otra referencia, implica también un juicio de valor. 
Finalmente, hay que tener en cuenta que en cada Comunidad existe ui número de personas distintos afectados por dicha diferencia de desarrollo, po lo que en cada caso hay que ponderar por el número de habitantes de cada un de ellas.

En definitiva, y siguiendo lo hasta aquí propuesto, se puede calcular para cad. año la participación que hipotéticamente hubiese tenido cada Comunidad en e FCI, de haberse seguido este criterio, según la fórmula que a continuación st representa:

$$
P P F C I^{\prime}=\frac{\left[\frac{P I B}{N^{\circ} H A B}-X\right] \cdot N^{\circ} H A B}{\sum_{j=1}^{17}\left[\frac{P I B(j)}{N^{0} H A B(j)}-X\right] \cdot N^{\circ} H A B(j)}
$$

siendo X el máximo de los PIB per cápita de las Comunidades Autónomas er cada año.

Con ello el Saldo Financiero Acumulado (hipótesis 2) de cada Comunidac vendría dado por la ecuación:

$$
S F A=\sum_{i=1988}^{1991}\left[P P F C I(i)-\operatorname{PPFCI}^{\prime}(i)\right] \cdot F C I(i) \cdot[1+s(i)]
$$

y con él quedaría expresada la pérdida o beneficio obtenido por cada Comunidad en el período considerado, en relación al hipotético reparto de FCI que se obtendría de haberse aplicado una concepción de la solidaridad y un criterio de reparto como los propuestos.

\section{LOS DATOS.}

La concreción de lo hasta aquí expuesto se presenta en los cuadros siguientes:

- Cuadro III: Incluye las cifras de Saldo Financiero Acumulado obtenidas para los ejercicios 1987 hasta I992, suponiendo que se hubiese mantenido constante el porcentaje de participación en el FCI obtenido en 1987 por 
cada Comunidad. Dicho saldo está calculado a pesetas de primeros de enero de 1992, según los datos de Índice de Precios de Consumo publicados por el Instituto Nacional de Estadística.

\section{CUADRO III}

\begin{tabular}{lccc}
\hline \multicolumn{1}{c}{ C.A. } & SFA/PFCI (1992) & SFA(mill. pts) & SFA/N H (pts./hab) \\
\hline Cataluña & $238,61 \%$ & $56.256,23$ & 9.241 \\
Murcia & $108,04 \%$ & $4.655,86$ & 4.484 \\
Canarias & $67,76 \%$ & $6.786,99$ & 4.636 \\
Galicia & $24,78 \%$ & $6.372,11$ & 2.300 \\
Andalucía & $13,59 \%$ & $8.010,09$ & 1.162 \\
Comunidad Valenciana & $-5,05 \%$ & $-614,80$ & -160 \\
Madrid & $-6,63 \%$ & $-716,39$ & -144 \\
Castilla-La Mancha & $-59,14 \%$ & $-8.417,47$ & -5.061 \\
Aragón & $-78,84 \%$ & $-3.209,93$ & -2.718 \\
Asturias & $-81,76 \%$ & $-4.165,79$ & -3.785 \\
La Rioja & $-102,15 \%$ & $-617,30$ & -2.340 \\
Castilla y León & $-105,61 \%$ & $-18.527,56$ & -7.242 \\
País Vasco & $-116,06 \%$ & $-12.031,45$ & -5.713 \\
Cantabria & $-124,55 \%$ & $-2.040,96$ & -3.870 \\
Baleares & $-136,92 \%$ & $-1.977,01$ & -2.813 \\
Extremadura & $-170,87 \%$ & $-24.256,38$ & -22.879 \\
Navarra & - & $-5.506,22$ & -10.624 \\
\hline
\end{tabular}

Fuente: Elaboración propia.

En dicho cuadro se ordenan las Comunidades según el porcentaje que representa dicho saldo sobre su participación en el FCI y la Compensación Transitoria de $1992^{8}$. Incluyéndose además el Saldo Financiero Acumulado en términos per cápita sobre la población de 1991.

- Cuadro IV y V: Presentan los resultados de reparto del FCI en porcentaje y en millones de pesetas, respectivamente, si se toma el criterio de reparto definido anteriormente, es decir la segunda hipótesis ${ }^{9}$.

8. Para entender el significado del porcentaje tomemos por el ejemplo el caso de Cataluña. El $238,61 \%$ significa que como consecuencia de la reforma. Cataluña ha obtenido fondos que representan casi 2,4 veces lo obtenido en el año 1992.

9. Para todos los años Baleares es la Comunidad de mayor PIB per cápita. 
La reforma del fondo de compensación interterritorial. Los costes. . .

CUADRO IV

\begin{tabular}{lrrrrrr}
\hline \multicolumn{1}{c}{ C.A. } & 1987 & 1988 & 1989 & 1990 & 1991 & 1992 \\
\hline Andalucía & 28,86 & 28,67 & 33,06 & 34,73 & 33,59 & 32,70 \\
Aragón & 2,34 & 2,33 & 2,37 & 2,30 & 2,35 & 2,43 \\
Asturias & 3,05 & 3,13 & 3,74 & 3,86 & 3,86 & 3,80 \\
Baleares & 0,00 & 0,00 & 0,00 & 0,00 & 0,00 & 0,00 \\
Canarias & 3,92 & 3,87 & 3,90 & 4,32 & 4,41 & 4,35 \\
Cantabria & 1,54 & 1,52 & 1,66 & 1,71 & 1,69 & 1,53 \\
Castilla León & 6,42 & 6,39 & 8,50 & 8,65 & 8,64 & 8,75 \\
Cas.-La Mancha & 8,06 & 7,92 & 6,70 & 6,96 & 6,87 & 6,80 \\
Cataluña & 7,66 & 7,72 & 3,00 & 1,84 & 2,71 & 3,50 \\
Valencia & 8,29 & 8,17 & 8,68 & 8,73 & 8,71 & 8,84 \\
Extremadura & 4,91 & 4,89 & 5,56 & 5,77 & 5,56 & 5,39 \\
Galicia & 10,45 & 10,47 & 10,72 & 11,01 & 10,80 & 10,54 \\
Madrid & 5,08 & 5,52 & 2,92 & 1,57 & 2,10 & 2,47 \\
Murcia & 3,49 & 3,54 & 4,06 & 4,19 & 4,11 & 4,07 \\
Navarra & 1,00 & 1,01 & 0,67 & 0,00 & 0,00 & 0,00 \\
País Vasco & 4,36 & 4,28 & 3,98 & 3,90 & 4,11 & 4,31 \\
La Rioja & 0,58 & 0,57 & 0,48 & 0,46 & 0,49 & 0,52 \\
TOTAL & 100,00 & 100,00 & 100,00 & 100,00 & 100,00 & 100,00 \\
\hline
\end{tabular}

Fuente: Elaboración propia.

Los datos utilizados para su cálculo: PIB per cápita y número de habitantes, han sido tomados de los Anexos 6, 7 y 8 de la revista Papeles de Economía Española, números 46, 51 y 55 de los años 1991, 1992 y 1993, respectivamente.

- Cuadro VI: Finalmente, este cuadro incluye el cálculo del Saldo Financierc Acumulado para Ios ejercicios 1987 a 1992, según las cifras obtenidas er los dos cuadros anteriores, expresándolo en pesetas de primeros de enerc de 1992.

Al igual que en el caso del cuadro III se incorpora ese mismo saldc como porcentaje de la participación en el FCI y la Compensación Transitoria en el ejercicio de 1992, y se ordenan las Comunidades en base a él. También se introduce la citada magnitud en términos per cápita. 


\section{CUADRO V}

(en millones de pesetas)

\begin{tabular}{lrrrrrr}
\hline \multicolumn{1}{c}{ C.A. } & 1987 & 1988 & 1989 & 1990 & 1991 & 1992 \\
\hline Andalucía & $40.442,27$ & $43.125,98$ & $67.358,71$ & $83.282,71$ & $86.454,95$ & $70.228,83$ \\
Aragón & $3.277,59$ & $3.503,19$ & $4.822,98$ & $5.525,12$ & $6.045,74$ & $5.217,11$ \\
Asturias & $4.269,70$ & $4.715,31$ & $7.624,90$ & $9.251,94$ & $9.930,38$ & $8.170,26$ \\
Baleares & 0,00 & 0,00 & 0,00 & 0,00 & 0,00 & 0,00 \\
Canarias & $5.487,40$ & $5.817,34$ & $7.945,53$ & $10.359,63$ & $11.351,40$ & $9.345,72$ \\
Cantabria & $2.153,67$ & $2.290,90$ & $3.378,44$ & $4.100,28$ & $4.339,19$ & $3.282,11$ \\
Castilla León & $8.997,30$ & $9.610,02$ & $17.315,70$ & $20.733,70$ & $22.239,45$ & $18.782,11$ \\
Cas.-La Mancha & $11.291,41$ & $11.919,56$ & $13.648,56$ & $16.883,85$ & $17.690,85$ & $14.601,90$ \\
Cataluña & $10.727,75$ & $11.612,37$ & $6.122,40$ & $4.414,00$ & $6.982,18$ & $7.516,83$ \\
C. Valenciana & $11.616,44$ & $12.297,12$ & $17.696,06$ & $20.932,38$ & $22.427,38$ & $18.974,03$ \\
Extremadura & $6.875,24$ & $7.351,48$ & $11.323,43$ & $13.847,43$ & $14.301,39$ & $11.565,72$ \\
Galicia & $14.640,38$ & $15.753,06$ & $21.840,86$ & $26.401,87$ & $27.792,51$ & $22.636,67$ \\
Madrid & $7.123,54$ & $8.304,94$ & $5.958,27$ & $3.766,27$ & $5.413,63$ & $5.295,43$ \\
Murcia & $4.897,36$ & $5.318,44$ & $8.266,26$ & $10.040,28$ & $10.576,64$ & $8.744,48$ \\
Navarra & $1.408,24$ & $1.519,61$ & $1.371,61$ & 0,00 & 0,00 & 0,00 \\
País Vasco & $6.106,03$ & $6.437,73$ & $8.115,96$ & $9.352,39$ & $10.578,06$ & $9.260,13$ \\
La Rioja & 815,97 & 858,26 & 971,41 & $1.110,13$ & $1.258,73$ & $1.115,10$ \\
TOTAL & $140.130,30$ & $150.435,30$ & $203.761,10$ & $239.802,00$ & $257.382,50$ & $214.736,70$ \\
\hline
\end{tabular}

Fuente: Elaboración propia.

Atendiendo a los datos presentados en los cuadros III y VI, representativos del Saldo Financiero Acumulado según las dos alternativas propuestas, y tomando como definición de Comunidad "pobre", aquella que en 1992 tiene un PIB per cápita inferior a la media española, se puede obtener las siguientes conclusiones:

1.- Existen dos Comunidades: Canarias y Galicia que presentan un SFA positivo en ambos casos y que, por consiguiente, han resultado beneficiadas en el proceso de redistribución asociado al reparto del FCI en el período considerado.

2.- Aparecen otras dos Comunidades: Castilla y León y Extremadura que si bien han perdido posiciones respecto a la situación de 1987, al presentar un SFA negativo según la hipótesis primera, por el contrario han resultado beneficiadas si tomamos como indicador el obtenido de la aplicación de la segunda hipótesis. 


\section{CUADRO VI}

\begin{tabular}{lccc}
\hline \multicolumn{1}{c}{ C.A. } & SFA/PFCI (1992) & SFA(mill. pts) & SFA/N H (pts./hal \\
\hline Baleares & $680,34 \%$ & $9.823,46$ & 13.818 \\
Cataluña & $352,94 \%$ & $83.209,43$ & 13.695 \\
Madrid & $270,76 \%$ & $29.251,89$ & 5.889 \\
Canarias & $212,01 \%$ & $21.235,53$ & 14.135 \\
Extremadura & $126,92 \%$ & $18.017,74$ & 16.931 \\
País Vasco & $87,98 \%$ & $9.120,96$ & 4.329 \\
Castilla y León & $39,16 \%$ & $6.870,77$ & 2.695 \\
Galicia & $24,31 \%$ & $6.251,72$ & 2.289 \\
Castilla-La Mancha & $-97,89 \%$ & $-13.933,59$ & -8.381 \\
Aragón & $-118,69 \%$ & $-4.832,53$ & -4.068 \\
Andalucía & $-123,06 \%$ & $-72.528,06$ & -10.402 \\
Comunidad Valenciana & $-262,63 \%$ & $-32.004,02$ & -8.279 \\
Asturias & $-398,17 \%$ & $-20.287,75$ & -18.583 \\
Murcia & $-540,48 \%$ & $-23.291,78$ & -22.156 \\
La Rioja & $-582,59 \%$ & $-3.520,58$ & -13.355 \\
Cantabria & $-709,40 \%$ & $-11.625,01$ & -22.034 \\
Navarra & - & $-1.758,17$ & -3.382 \\
\hline
\end{tabular}

Fuente: Elaboración propia.

3.- Un tercer grupo de Comunidades, el formado por Murcia y Andalucía, presenta una situación inversa a la anterior, ya que a pesar de no haber sido perjudicadas en el proceso, considerando su situación en 1987, sin embargo sí son perjudicadas si se interpreta la solidaridad interterritorial en la forma propuesta en este trabajo.

4.- Finalmente, las Comunidades de Cantabria, Asturias y Castilla-La Mancha, obtienen un SFA negativo en los dos supuestos, por lo que se las puede declarar claras perdedoras del proceso transformación del FCI.

Por lo que se refiere a las Comunidades con PIB per cápita por encima de la media nacional:

1.- Las Comunidades de Aragón, Valenciana, y de La Rioja aparecen como perdedoras, pues en ambos casos los SFA son negativos ${ }^{10}$.

10. En el caso de Navarra lo dejamos al margen por haber quedado definitivamente excluida del FCI y de la Compensación Transitoria, a partir del año 1990. 
CUADRO VII

\begin{tabular}{lrr}
\hline \multicolumn{1}{c}{ C.A. } & \% PIBPP & PIBPP (1991) \\
\hline Baleares & $137,93 \%$ & 2.135 .225 \\
Madrid & $130,70 \%$ & 2.023 .265 \\
Cataluña & $129,54 \%$ & 2.005 .302 \\
Navarra & $116,69 \%$ & 1.806 .340 \\
La Rioja & $109,23 \%$ & 1.690 .932 \\
Aragón & $108,13 \%$ & 1.673 .914 \\
País Vasco & $108,11 \%$ & 1.673 .557 \\
Comunidad Valenciana & $104,63 \%$ & 1.619 .684 \\
Canarias & $95,72 \%$ & 1.481 .800 \\
Cantabria & $95,72 \%$ & 1.481 .800 \\
Castilla y León & $87,95 \%$ & 1.361 .496 \\
Asturias & $87,15 \%$ & 1.349 .165 \\
Galicia & $81,69 \%$ & 1.264 .544 \\
Murcia & $81,49 \%$ & 1.261 .502 \\
Castilla-La Mancha & $78,34 \%$ & 1.212 .720 \\
Andalucía & $69,59 \%$ & 1.077 .246 \\
Extremadura & $64,19 \%$ & 993.656 \\
ESPAÑA & $100,00 \%$ & 1.548 .033 \\
\hline
\end{tabular}

Fuente: Anexo 8, Papeles de Economía Española, 55, 1993.

2.- Sin embargo, existe un grupo, el formado por las Comunidades de Baleares, Madrid y el País Vasco, que si bien han perdido fondos en relación a 1987, obtienen significativas ganancias si consideramos la segunda hipótesis de reparto.

3.- Finalmente el grupo de los ganadores para ambos criterios, está formado por una única región: Cataluña, que ha obtenido sustanciosos fondos adicionales con la reforma, más de cincuentamil millones según la primera hipótesis, y más de ochentamil según la segunda.

\section{EL CASO DE ANDALUCÍA.}

Andalucía, probablemente por la importancia política de la región, ha resuelto favorecida en relación a la participación que tenía en 1987, obteniendo un SFA de más de quincemil millones a lo largo de los tres últimos años considerados, 
que compensan las pérdidas acumuladas durante el período de ausencia de 1 . Compensación Transitoria (Ver cuadro VIII). Y que representa una ganancia medi de dos puntos de porcentaje de participación, como aparece en la fila nueve.

\section{CUADRO VIII}

Datos desagregados para Andalucía

\begin{tabular}{lrrrrrr}
\hline \multicolumn{1}{c}{ C.A. } & 1987 & \multicolumn{1}{c}{1988} & 1989 & 1990 & 1991 & \multicolumn{1}{c}{1992} \\
\hline FCI & $35.771,40$ & $36.927,60$ & $49.855,60$ & $65.768,00$ & $71.203,90$ & $58.935,00$ \\
FCI (hipo. 1) & $35.771,40$ & $38.401,98$ & $52.014,84$ & $61.214,84$ & $65.702,65$ & $54.816,36$ \\
Diferencia & 0,00 & $-1.474,38$ & $-2.159,24$ & $4.553,16$ & $5.501,25$ & $4.118,64$ \\
FCI (hipo.2) & $40.442,27$ & $43.125,98$ & $67.358,71$ & $83.282,71$ & $86.454,95$ & $70.228,83$ \\
Diferencia & $-4.670,87$ & $-6.198,38$ & $-17.503,11$ & $-17.514,71$ & $-15.251,05$ & $-11.293,83$ \\
PPFCI & 25,53 & 24,55 & 24,47 & 27,43 & 27,66 & 27,45 \\
PPFCI (h. 1) & 25,53 & 25,53 & 25,53 & 25,53 & 25,53 & 25,53 \\
Diferencia & 0,00 & $-0,98$ & $-1,06$ & 1,90 & 2,13 & 1,92 \\
PPFCI (h. 2) & 28,86 & 28,67 & 33,06 & 34,73 & 33,59 & 32,70 \\
Diferencia & $-3,33$ & $-4,12$ & $-8,59$ & $-7,30$ & $-5,93$ & $-5,25$ \\
\hline
\end{tabular}

Fuente: Elaboración propia.

Si bien dicha ganancia es la menor de las obtenidas por el grupo de Ia: Comunidades beneficiadas, que como mínimo duplican la ganancia (Galicia) c la multiplican por 18 (Cataluña), en relación a su participación en el año 199; (ver columna segunda del cuadro III).

Por el contrario, si tomamos como referencia el reparto derivado de li aplicación de la segunda hipótesis, Andalucía aparece como clara perdedora de proceso, pues desde el primer momento tiene una pérdida que oscila entre : y 8 puntos de participación (fila 11 del cuadro VIII). Bajo este supuesto, e sistema de Compensación transitoria, en lugar de mejorar, agudiza lí insolidaridad del reparto. Lo cual se observa claramente si comparamos lo: datos obtenidos para Andalucía, con los que se obtienen para Cataluña, y que aparecen en el cuadro IX. 
CUADRO IX

Datos desagregados para Cataluña

\begin{tabular}{lrrrrrr}
\hline \multicolumn{1}{c}{ C.A. } & 1987 & \multicolumn{1}{c}{1988} & 1989 & 1990 & 1991 & \multicolumn{1}{c}{1992} \\
\hline FCI & $10.044,70$ & $14.768,80$ & $23.892,50$ & $26.282,00$ & $28.259,80$ & $23.576,20$ \\
FCI (hipo. 1) & $10.044,70$ & $10.783,37$ & $14.605,83$ & $17.189,28$ & $18.449,47$ & $15.392,57$ \\
Diferencia & 0,00 & $3.985,43$ & $9.286,67$ & $9.092,72$ & $9.810,33$ & $8.183,63$ \\
FCI (hipo.2) & $10.727,75$ & $11.612,37$ & $6.122,40$ & $4.414,00$ & $6.982,18$ & $7.516,83$ \\
Diferencia & $-683,05$ & $3.156,43$ & $17.770,10$ & $21.868,00$ & $21.277,62$ & $16.059,37$ \\
PPFCI & 7,17 & 9,82 & 11,73 & 10,96 & 10,98 & 10,98 \\
PPFCI (h. 1) & 7,17 & 7,17 & 7,17 & 7,17 & 7,17 & 7,17 \\
Diferencia & 0,00 & 2,65 & 4,56 & 3,79 & 3,81 & 3,81 \\
PPFCI (h. 2) & 7,66 & 7,72 & 3,00 & 1,48 & 2,71 & 3,50 \\
Diferencia & $-0,49$ & 2,10 & 8,73 & 9,48 & 8,27 & 7,48 \\
\hline
\end{tabular}

Fuente: Elaboración propia.

\section{RESUMEN DE CONCLUSIÓN.}

A resueltas de los datos, la conclusiones no pueden ser otras que las siguientes:

a) Las reformas del FCI no le han orientado al cumplimiento de su función de solidaridad, ya que el FCI ha actuado como un mecanismo de compensación financiera de la fuente básica de financiación incondicionada. Prueba de ello son los valores de ponderación aplicados a los criterios de reparto, las interpretaciones forzadas del mínimo de dotación del fondo, y la ausencia de un contenido claro del concepto de solidaridad.

b) El resultado cuantitativo de las reformas, pone de manifiesto la paradójica situación de que la región claramente beneficiada, en relación a su posición en 1987, es Cataluña, a pesar de ser la tercera región española en PIB per cápita. Lo que acrecienta aún más la impresión de que difícilmente se puede entender que sea la solidaridad el principio orientador de dichas reformas. 
c) Cuando se toma como objetivo del reparto del FCI la minoración de la diferencias de los PIB per cápita regionales y se compara con el repartc real, se pone claramente de manifiesto la inconsistencia del actual FCI cor. dicho objetivo, y como el FCI probablemente esté contribuyendo a acrecentar más las ya de por si elevadas diferencias.

d) Finalmente, por lo que se refiere a Andalucía, probablemente por su importancia política, ha conseguido enmendar los efectos iniciales de la reforma, al menos durante el tiempo que ha durado la Compensación Transitoria. Sin embargo no ha podido evitar las insolidarias interpretaciones de la ley en cuanto a la dotación del fondo, ni ha inducido a la interpretación de la solidaridad como "redistribución para el desarrollo". Una fórmula como la propuesta en el presente artículo le hubiese supuesto unos ingresos adicionales de más de setenta y dos mil millones de pesetas, y una clara vocación solidaria del FCI para eI futuro. Una vocación que permita repartir justamente los costes y beneficios de la solidaridad.

\section{BIBLIOGRAFÍA}

ALCAIDE INCHAUSTI, J., CUADRO, J. R. y FUENTES QUINTANA, E. (1990): El desarrollo económico español y la España desigual de las Autonomías. Papeles de Economía Española, 45, págs. 2-61.

BIEHL, D. (1988): Las infraestructuras y el desarrollo regional. Papeles de Economía Española, 35, págs. 293-310. .

BOSCH, A. y ESCRIBANO, C. (1988): Las necesidades de gastos de las Comunidades Autónomas. Instituto de Estudios Fiscales (Ed.), págs. 209-269.

CALSAMIGLIA, X. (1988): Descentralización del gasto público y financiación autonómica. Una valoración del sistema español. Documentos de trabajo I. A. E., A01.

CALSAMIGLIA, X. (1990): La financiación de las Comunidades Autónomas y el principio de solidaridad. Economía Pública, 6, págs. 3-44.

CASTELLS, A. (1988): Hacienda Autonómica. Una perspectiva de federalismo fiscal. Ariel, Barcelona.

CASTELLS, A., COSTA, M., PERULLES, J. y SICART, F. (1986): La financiación de las Comunidades Autónomas: el período transitorio y la revisión del sistema. Secretaría de Estado de Hacienda, Madrid.

FALCÓN Y TELLA, R. (1990): El fondo de Compensación Interteritorial. Seminario sobre Financiación de las Comunidades Autónomas en España. U. I. M. P., Valencia. 
GARCÍA AÑOVEROS, J. (1986a): ¿Crisis en las haciendas autonómicas?. Hacienda Pública Española, 101, págs. 367-374.

GARCÍA AÑOVEROS, J. (1986b): Informe sobre modificación del sistema de financiación de las

Comunidades Autónomas. Hacienda Pública Española, 101, págs. 374-403.

HIERRO RECIO, L. A. (1992): Análisis económico del Estado descentralizado y de su financiación. Propuesta para el caso español. Tesis Doctoral, Universidad de Sevilla.

IGLESIAS SUÁREZ, A., PUY FRAGA, P. y RODRÍGUEZ PADRÓN, C. (1984): Comentario en tomo a la financiación de la autonomía en Galicia (ingresos). Hacienda Pública Española, 89, págs. 127-144.

INSTITUTO DE ESTUDIOS FISCALES (Ed.) (1988): Cinco estudios sobre la financiación autonómica. I. E. F., Madrid.

LÓPEZ NIETO, A. y FERNÁNDEZ RODRÍGUEZ, F. (1983): Sobre el significado económico del Fondo Compensación Interterritorial. Hacienda Pública Española, 84, págs. 217-249.

MELGUZZO SÁNCHEZ, A. (1988): El Fondo de Compensación Interterritorial. Papeles de Economía Española, 35, págs. 354-376.

PUJOL, J. (1989): Aspectos de la hacienda autonómica y federal en el contexto europeo. Papeles de Economía Española, 41, págs. 279-285.

RUIZ-CASTILLO, J. y SEBASTIAN, C. (1985): El sistema de financiación autonómica: críticas y alternativas. Instituto de Estudios Fiscales (Ed.) (1988), págs. 117-208.

TORRES COBO, F. (1984): La financiación de las Comunidades Autónomas en los Presupuestos Generales del Estado. Hacienda Pública Española, 90, págs. 277-296.

TORRES COBO, F. (1990): El sistema de financiación de las Comunidades Autónomas. Instituto de Estudios Fiscales, Madrid.

UTRILlA DE LA HOZ, A. (1991): Análisis de los efectos redistributivos del Fondo de Compensación Interterritorial. Documentos de Trabajo de F. I. E. S., 72/1991. 